\title{
Análise de parcimônia de endemismo de membracídeos neotropicais (Hemiptera, Membracidae, Hoplophorionini)
}

\author{
Ângela Goldani ${ }^{1}$ \\ Augusto Ferrari ${ }^{1}$ \\ Gervásio Silva Carvalho ${ }^{1}$ \\ Antônio José Creão-Duarte ${ }^{2}$
}

\begin{abstract}
Parsimony Analysis of Endemicity based on neotropicals treehoppers (Hemiptera, Membracidae, Hoplophorionini). The Parsimony Analysis of Endemicity (PAE) consists in an important tool of the historical biogeography which has the purpose of detecting distribution patterns of organisms and the relationships among areas of endemism. Insect distribution data included in Membracidae-Hoplophorionini occurring in the American Continent were used in the present research in order to establish the relationships among eight predetermined areas of endemism which are: 1) Mexico, 2) Greater Antilles, 3) Central America, 4) North Andes, 5) Central Andes, 6) South Andes, 7) Amazon, e 8) South East Brazil. The data matrix was based on the presence (coded as 1 ) and abscence ( 0$)$ of taxa in the areas. The analysis was performed with the use of computer program Hennig86 (FARRIS 1988) applying the comands ie (implicit enumeration), and $\mathbf{x s} \mathbf{w}$ (successive weighting). Resulted in the cladogram (A2,((A6,(A1,(A3,(A4,A5)))),(A7,A8))) with 236 steps, consistency index $=0.94$ and retention index $=0.86$. Areas 7 and 8 follow a subtropical and tropical vegetation pattern made up respectively by pluvial forests and groves. The remaining areas (except 2 ) agree with mountainous vegetation of the Andes pattern and groves throughout the extention.

KEY WORDS. Biogeography, Neotropical region, distribution, areas of endemism, treehoppers
\end{abstract}

A região Neotropical é detentora de uma alta biodiversidade, e as razões incluem desde fatores climáticos a biogeográficos. Biogeógrafos são levados a usar todos os dados distribucionais conhecidos para construir uma hipótese mais parcimoniosa das distribuições recentes dos táxons (NELSON 1969), tentando responder a questão "por que os táxons estão distribuídos onde ocorrem atualmente?" (PLATNICK \& NELSON 1978). O endemismo é uma das mais significantes características da distribuição biogeográfica (ROSEN 1988). Como determinar as relações históricas entre as áreas de endemismo é uma das questões principais da biogeografia histórica (NELSON \& PlATNICK 1981; HumPHRIES \& PARENTI 1999).

Estudos biogeográficos são dependentes de dados concretos sobre a distribuição geográfica dos grupos selecionados para análise. A visão de como a endemicidade de espécies está distribuída dentro de uma região é muito importante e requer busca qualitativa e quantitativa de dados. O método de Análise de Parcimônia de Endemismo

1) Faculdade de Biociências, Pontifícia Universidade Católica do Rio Grande do Sul. Avenida Ipiranga 6681, 90619-900 Porto Alegre, Rio Grande do Sul, Brasil.

2) Departamento de Sistemática e Ecologia, Universidade Federal da Paraíba. Campus I, 58059-900 João Pessoa, Paraíba, Brasil. 
(PAE) é considerado uma ferramenta da biogeografia histórica que permite descobrir os padrões naturais de distribuição dos organismos (ROSEN 1988; ROSEN \& SMITH 1988). Um cladograma PAE pode contribuir na classificação de biotas de acordo com a distribuição do grupo em estudo, bem como favorecer na interpretação das condições ecológicas contemporâneas de cada área de endemismo estabelecida e as relações históricas entre elas. O reconhecimento de áreas de endemismo é um passo importante para o estudo biogeográfico de qualquer táxon. Além da importância para o estudo biogeográfico, a delimitação de áreas de endemismo pode contribuir em projetos de conservação da natureza (MORRONE 2000).

RON (2000) acredita que o PAE (ou outras técnicas da biogeografia cladística) deve ser aplicado somente para os organismos que tem sua capacidade de dispersão limitada e que sofreram especiação através do modelo vicariante. Os insetos, apesar de muitos terem distribuição cosmopolita, podem também ocorrer em áreas restritas, seguindo os padrões ecológicos e climáticos diversificados existentes, o que permite a utilização do método PAE.

Muitos autores tem usado o método para postular relações entre áreas de endemismo, e também para comparar seus resultados com outros métodos biogeográficos.

MORRONE (1994) propôs a aplicação de um algoritmo de simplicidade para delimitar áreas de endemismo e para isso utilizou, como unidades de análise, quadrículas a partir de linhas geográficas latitudinais e longitudinais, reunidas pela presença compartilhada dos táxons. Seu método pode ser referenciado como Análise de Parcimônia de Endemismo baseado em quadrículas.

Morrone \& LOPRETTO (1995) usaram o PAE na região sul da América do Sul e identificaram nove áreas de endemismo de Decapoda e três relações entre as áreas foram estabelecidas.

POSADAS (1996) aplicou o PAE na Terra do Fogo com dados de distribuição de plantas vasculares usando 52 quadrículas como Unidades Geográficas Operacionais (OGUs - Operative Geografic Unit). Segundo o cladograma de consenso resultante, dois grandes grupos foram determinados e subdivididos em áreas de endemismo menores.

SILVA \& OREN (1996) utilizaram o PAE em um estudo da biogeografia Amazônica com dados de distribuição de primatas da região. Neste trabalho foram usadas como OGUs 14 regiões interfluviais da bacia Amazônica, referenciadas como possíveis barreiras biogeográficas. CRACRAFT (1985) trabalhou com a mesma região utilizando dados de distribuição de aves e os resultados foram comparados com os de primatas, apresentando boa correspondência.

VEGA et al. (1999) trabalharam com o PAE na distribuição de 1267 espécies de plantas vasculares nos bosques mesófilos de montanhas mexicanas. Foram utilizadas como OGUs 24 províncias florestais, e cinco grandes áreas de endemismo foram estabelecidas.

RON (2000) testou hipóteses da biogeografia dos anuros neotropicais de florestas úmidas usando o PAE em 14 localidades obtendo forte congruência com análises utilizando lagartos (ÁVILA-PIRES 1995) e primatas (SILVA \& OREN 1996). 
BISCONTI et al. (2001) usaram dados de distribuição de répteis, aves e plantas das Ilhas Galápagos, verificando diferenças nos padrões de distribuição dos seres vivos nas ilhas e que isso pode estar relacionado com eventos biogeográficos ocorridos em épocas e locais diferentes do arquipélago.

GARCÍA-BARROS et al. (2002) estudaram a distribuição de 480 espécies de plantas e animais aplicando o PAE e análise de compatibilidade na Península Ibérica e nas Ilhas Baleares, baseando-se em dados de distribuição plotados em quadrículas de $100 \mathrm{~km}^{2}$. Utilizaram também técnica de eliminação progressiva (PAE-PCE) de caracteres (espécies) objetivando minimizar alguns defeitos potenciais do método. O procedimento proposto (PAE-PCE) provou ser útil na identificação de áreas de endemismo que não teriam sido detectadas quando utilizada somente o PAE.

No presente trabalho o método de Análise de Parcimônia de Endemismo (PAE) foi utilizado para gerar um cladograma de relacionamento entre as áreas de endemismo pré-determinadas baseadas em dados de distribuição de Membracídae. As áreas de endemismo se delimitam seguindo o conceito de PLATNICK (1991) que as classifica como uma região abrangendo um limite distribucional congruente de duas ou mais espécies.

\section{MATERIAL E MÉTODOS}

As análises foram baseadas em dados de distribuição obtidos em CREÃODuarte (1997), CreÃo-Duarte \& Sakakibara (1996a,b, 1997) e Metcalf \& WADE (1965).

O método usado foi proposto por CRACRAFT (1991), o qual difere do método original de ROSEN (1988) por utilizar como unidades geográficas operacionais (OGUs) áreas de endemismo pré-definidas, de acordo com a sobreposição de duas ou mais espécies em uma região. Como preconiza o método, a matriz (Tab. I) foi construída utilizando-se as áreas de endemismo pré-estabelecidas por táxons ali encontrados, isto é, 29 espécies além de três gêneros (Ochropepla Stål, 1869, Metcalfiella Goding, 1929 e Platycotis Stål, 1869) incluídos em Membracidae - Hoplophorionini. A presença do táxon, sempre considerado como monofilético, foi codificada como 1 e ausência como 0. Uma área hipotética considerada como primitiva e codificada como 0 para todos os táxons, foi incluída objetivando polarização. A matriz foi analisada com o programa computacional Hennig86 (FARRIS 1988) aplicando o comando ie (enumeração implícita), e, em análise sucessiva, o comando xs $\mathbf{w}$ (pesos sucessivos). Na matriz, quando comparada com a de análise de táxons, as áreas substituem os táxons, e os táxons os caracteres (ROSEN 1988; MYERS 1991; MORRONE 1994; MORRONE \& CRISCI 1995; POSADAS 1996).

\section{RESULTADOS}

Da análise distribucional dos dados de Hoplophorionini, resultaram oito áreas de endemismo (Fig. 1) referenciadas como: A1 - México: região sul do México $\left(25^{\circ}-15^{\circ} \mathrm{N}, 105^{\circ}-100^{\circ} \mathrm{W}\right) ; \mathrm{A} 2-$ Grandes Antilhas: sul da Flórida e Cuba $\left(30^{\circ}-20^{\circ} \mathrm{N}\right.$, $\left.85^{\circ}-75^{\circ} \mathrm{W}\right)$; A3 - América Central $\left(15^{\circ}-5^{\circ} \mathrm{N}, 90^{\circ}-80^{\circ} \mathrm{W}\right)$; A4 - Andes Norte: Venezuela, Colômbia, Equador e Peru $\left(10^{\circ} \mathrm{N}-10^{\circ} \mathrm{S}, 80^{\circ}-70^{\circ} \mathrm{W}\right)$; A5 - Andes Central: sul do Peru e região ocidental da Bolívia $\left(10^{\circ}-15^{\circ} \mathrm{S}, 75^{\circ}-70^{\circ} \mathrm{W}\right)$; A6 - Andes Sul: norte

Revta bras. Zool. 19 (Supl. 2): 187 - 193, 2002 


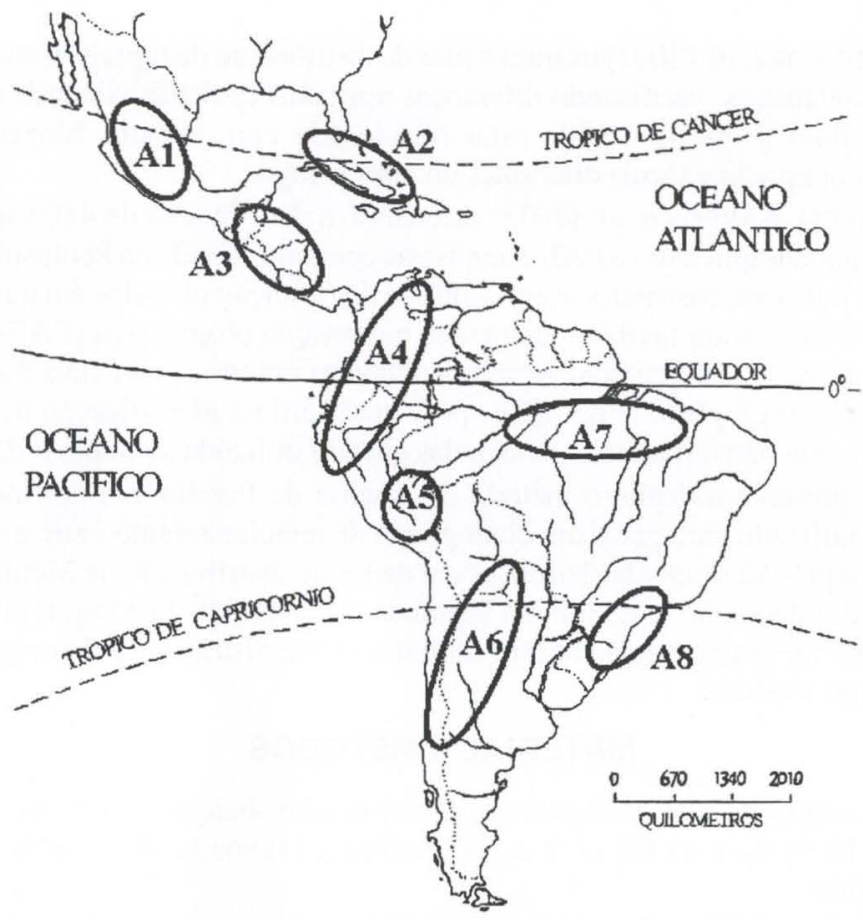

Fig.1. Mapa da Região Neotropical com as oito áreas de endemismo estabelecidas.

Tabela I. Matriz de dados da distribuição de 29 espécies e três gêneros de membracídeos em oito áreas de endemismo da Região Neotropical.

\begin{tabular}{|c|c|c|c|c|c|c|c|c|c|c|c|c|c|c|c|c|c|c|c|c|c|c|c|c|c|c|c|c|c|c|c|c|}
\hline eas Táxon & 1 & 2 & 3 & 4 & 5 & 6 & 7 & 8 & 9 & 0 & 1 & 2 & 3 & 4 & 5 & 6 & 7 & 8 & 9 & $\begin{array}{l}2 \\
0\end{array}$ & 1 & 2 & 3 & 4 & 5 & 6 & 7 & 8 & 9 & $\begin{array}{l}3 \\
0\end{array}$ & 1 & 2 \\
\hline$O G$ & 0 & 0 & 0 & 0 & 0 & 0 & 0 & 0 & 0 & 0 & 0 & 0 & 0 & 0 & 0 & 0 & 0 & 0 & 0 & 0 & 0 & 0 & 0 & 0 & 0 & 0 & 0 & 0 & 0 & 0 & 0 & 0 \\
\hline $\mathrm{A} 1$ & 0 & 0 & 0 & 0 & 0 & 0 & 0 & 0 & 0 & 0 & 0 & 0 & 0 & 0 & 0 & 0 & 0 & 0 & 0 & 0 & 0 & 0 & 0 & 0 & 0 & 1 & 1 & 0 & 1 & 1 & 1 & 1 \\
\hline ? & 0 & 0 & 0 & 0 & 0 & 0 & 0 & 0 & 0 & 0 & 0 & 0 & 0 & 0 & 0 & 0 & 0 & 0 & 0 & 0 & 0 & 0 & 1 & 0 & 0 & 0 & 1 & 0 & 0 & 0 & 0 & 1 \\
\hline $\mathrm{A} 3$ & 0 & 0 & 0 & 0 & 0 & 0 & 0 & 0 & 0 & 0 & 1 & 1 & 0 & 0 & 0 & 0 & 0 & 0 & 0 & 1 & 1 & 0 & 0 & 1 & 0 & 0 & 1 & 0 & 1 & 1 & 1 & 1 \\
\hline $\mathrm{A} 4$ & 1 & 1 & 0 & 1 & 1 & 1 & 1 & 0 & 1 & 1 & 1 & 1 & 0 & 0 & 1 & 0 & 0 & 0 & 0 & 0 & 0 & 1 & 0 & 1 & 1 & 0 & 1 & 1 & 1 & 1 & 1 & 0 \\
\hline A5 & 1 & 0 & 0 & 0 & 0 & 0 & 0 & 0 & 0 & 1 & 1 & 0 & 0 & 0 & 0 & 0 & 0 & 0 & 0 & 0 & 0 & 0 & 0 & 0 & 1 & 0 & 0 & 0 & 1 & 0 & 0 & 0 \\
\hline A6 & 0 & 0 & 1 & 0 & 0 & 0 & 0 & 1 & 0 & 0 & 0 & 0 & 0 & 0 & 0 & 0 & 0 & 0 & 0 & 0 & 0 & 0 & 0 & 0 & 0 & 0 & 0 & 1 & 0 & 1 & 1 & 0 \\
\hline A7 & 0 & 0 & 0 & 0 & 0 & 0 & 0 & 0 & 0 & 0 & 0 & 0 & 0 & 0 & 1 & 1 & 1 & 1 & 1 & 1 & 1 & 0 & 0 & 0 & 0 & 0 & 0 & 0 & 0 & 0 & 1 & 1 \\
\hline A8 & 0 & 0 & 0 & 0 & 0 & 0 & 0 & 0 & 0 & 0 & 0 & 0 & 1 & 1 & 1 & 1 & 0 & 0 & 1 & 0 & 0 & 0 & 0 & 1 & 0 & 0 & 0 & 0 & 1 & 0 & 1 & 1 \\
\hline
\end{tabular}

Relação dos táxons: 1) Alchisme pinguicornis, 2) Alchisme bos, 3) Alchisme salta, 4) Alchisme deflexa, 5) Alchisme fastidiosa, 6) Alchisme grossa, 7) Alchisme inermis, 8) Alchisme rubrocostata, 9) Alchisme tridentata, 10) Alchisme ustulata, 11) Alchisme virescens, 12) Alchisme apicalis, 13) Alchisme frontomaculata, 14) Alchisme obscura, 15) Alchisme testacea, 16) Alchisme turrita, 17) Potnia jacula, 18) Potnia gladiator, 19) Potnia venosa, 20) Potnia brevicornis, 21) Potnia granadensis, 22) Potnia inca, 23) Stalotypa fairmairi, 24) Umbonia ataliba, 25) Umbonia lativitta, 26) Umbonia reclinata, 27) Umbonia crassicornis, 28) Umbonia pyramidalis, 29) Umbonia spinosa, 30) Ochropepla spp., 31) Metcalfiella spp., 32) Platycotis spp. 
e centro do Chile, sul da Bolívia e região ocidental da Argentina $\left(15^{\circ}-40^{\circ} \mathrm{S}\right.$, $\left.75^{\circ}-65^{\circ} \mathrm{W}\right)$; A7 - Amazônia: região norte do Brasil $\left(0-5^{\circ} \mathrm{S}, 65^{\circ}-45^{\circ} \mathrm{W}\right)$ e A8 - Brasil Sudeste-Sul $\left(20^{\circ}-30^{\circ} \mathrm{S}, 55^{\circ}-40^{\circ} \mathrm{W}\right)$.

Dos trinta e dois táxons utilizados na análise, a maior diversidade restringe-se às áreas América Central e Andes Norte.

Da análise cladística resultou um único cladograma com 236 passos, índice de consistência 0,94 e índice de retenção 0,86 (Fig. 2).

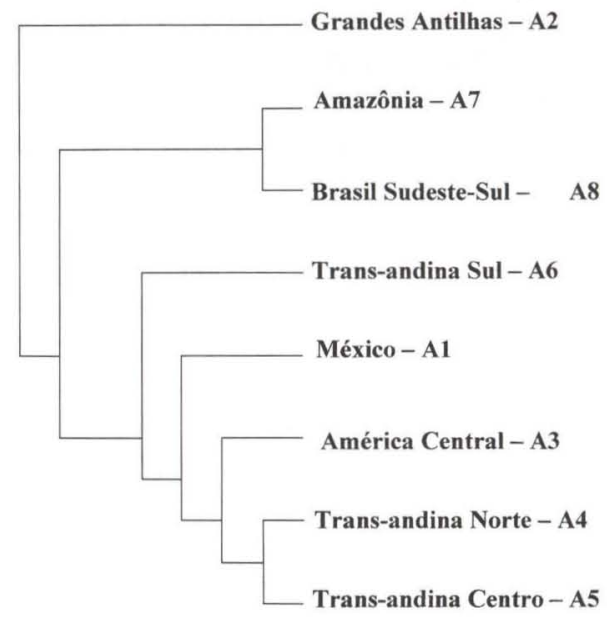

Fig. 2. Cladograma resultante com 236 passos, índice de consistência 0,94 e índice de retenção 0,86 .

\section{DISCUSSÃO}

Os membracídeos são insetos capazes de habitar regiões ecológicas bastante diversificadas. A área A2 está inserida em uma região de pradarias e savanas inundadas e ficou isolada no cladograma em posição basal, corroborando outros trabalhos baseados em dados distribucionais de táxons diferentes. O cladograma sugere que a relação das áreas A7 e A8 compartilham um padrão fisiográfico semelhante de vegetação tropical e subtropical formado por florestas pluviais e bosques, apesar de afastadas geograficamente, pela interposição basicamente de vegetação de cerrado. As demais áreas de endemismo apresentam um padrão faunístico e florístico diversificado, típico da região ocidental do continente americano. A explicação ecológica e fisiográfica torna-se difícil por ser uma grande área coberta pelos mais diversos padrões vegetais e climáticos, porém é certo afirmar que a ecologia influencia muito a distribuição da fauna entomológica. A área A1 caracteriza-se por abrigar florestas de coníferas, desertos e áreas arbustivas, além de regiões bastante úmidas. A área A3 é tipicamente florestal, mas com algumas pequenas regiões formadas por manguezais. As áreas A4, A5 e A6 apresentam características de bosques tropicais, subtropicais, temperados, de alta montanha, pradarias, savanas e até mesmo regiões bastante secas com flora xerófila típica de 
deserto. O clima das áreas de endemismo segue o padrão tropical-temperado característico da região Neotropical, porém com variações de clima frio de montanha presentes nas áreas $\mathrm{A} 4, \mathrm{~A} 5 \mathrm{e} \mathrm{A} 6$. O isolamento da região montanhosa da Cordilheira dos Andes (A4, A5, A6) em relação às florestas tropicais pode ter sido decisivo no aparecimento de áreas de endemismo.

Algumas espécies corroboraram sinapomorfias geográficas. Stalotypa fairmairei (Guérin-Méneville, 1856) ocorreu isolada na área A2. Duas espécies, Alchisme turrita (Germar, 1835) e Potnia venosa (Germar, 1821), justificam o agrupamento das áreas A7 e A8. Alchisme pinguicornis Funkhouser, 1940, Alchisme ustulata (Fairmaire, 1846) e Umbonia lativitta Walker, 1851 justificam o agrupamento das áreas A4 e A5. Umbonia spinosa (Fabricius, 1775) provavelmente tem uma capacidade de dispersão maior por ocorrer nas áreas A1, A3, A4 e A5, e estar presente também na área A8. As espécies de Metcalfiella estão presentes em todas as áreas, exceto A2 e A5, demonstrando uma possível falta de coleta. As espécies de Ochropepla justificam o ramo $(\mathrm{A} 6,(\mathrm{~A} 1,(\mathrm{~A} 3,(\mathrm{~A} 4, \mathrm{~A} 5))))$, mas ausentes na área $\mathrm{A} 5$.

As relações resultantes no cladograma corroboram a suposição de que a área A2 parece ter sofrido uma separação bem anterior à região Neotropical continental, além do isolamento produzido pela formação andina; de um lado o agrupamento oriental (A7 e A8) no escudo brasileiro e de outro o ocidental, o qual resultou um conjunto onde a área mais ao sul (A6), situa-se basalmente com as demais em seqüência do norte para o sul.

A interpretação histórica do cladograma resultante poderá ser melhor evidenciada se outros dados de distribuição, de diferentes grupos de organismos, forem utilizados, além de outros métodos da biogeografia cladística, contribuindo para um melhor entendimento e relacionamento das áreas de endemismo propostas.

\section{REFERÊNCIAS BIBLIOGRÁFICAS}

ÁviLA-PIRES, T.C.S. 1995. Lizards of Brazilian Amazonia (Reptilia: Squamata). Zool. Verh., Leiden, 299: 1-706. [1-11; 599-617].

Bisconti, M.; W. Landini; G. Bianucci; G. Cantalamessa; G. Carnevale; L. Ragaini \& G. Valleri. 2001. Biogeografic relationships of the Galapagos terrestrial biota: parsimony analysis of endemicity based on reptiles, land birds and Scalesia land plants. Jour. Biogeogr. 28: 495-510.

CRACRAFT, J. 1985. Historical biogeography and patterns of differentiation within the South American avifauna: Areas of endemism. Ornithol. Monogr. 36: 49-84.

1991. Patterns of diversification within continental biotas: Hierarquical congruence among the areas of endemism of Australian vertebrates. Aust. Syst. Bot., Collingwood, 4: 211-227.

CreÃo-Duarte, A.J. 1997. Novos Gêneros e Espécies de Hoplophorionini (Hemiptera, Membracidae, Membracinae). Revta bras. Zool. 14 (2): 417-423.

CReÃo-Duarte, A.J. \& A.M. SaKakibara. 1996a. Revisão do gênero Umbonia Burmeister (Homoptera, Membracidae, Membracinae, Hoplophorionini). Revta bras. Zool. 13 (4): 973-994.

- 1996b. Revisão do gênero Potnia Stål (Homoptera, Membracidae, Membracinae, Hoplophorionini). Revta bras. Zool. 13 (4): 1001-1021.

— 1997. Revisão de Alchisme Kirkaldy (Hemiptera, Membracinae, Hoplophorionini). Revta bras. Zool. 14 (2):425-472.

FARRIS, J.S. 1988. Hennig86 reference. Version 1.5. New York, Published by the autor, Port Jefferson Station.

García-Barros, E.; P. Gurrea; M.J. Luciáñez; J.M. Cano; M.L. Munguira; J.C. Moreno; H. Sainz; 
M.J. SANZ \& J.C. SiMón. 2002. Parsimony analysis of endemicity and its application to animal and plant geographical distributions in the Ibero-Balearic region (western Mediterranean). Jour. Biogeogr. 29: 109-124.

HumpHries, C.J. \& L.R. PARENTI. 1999. Cladistic biogeography. Oxford: Oxford Univ. Press, XI+187p. MetCALF, Z.P. \& V. WADE. 1965. General Catalogue of the Homoptera. A supplement to fascicle I-Membracidae of the General Catalogue of the Hemiptera. Membracoidea. Sec. II. Raleigh, North Carolina State Univ, p. 745-1552.

Morrone, J.J. 1994. On the identification of areas of endemism. Syst. Biol. 43: 438-441. 2000. La importancia de los atlas biogeográficos para la conservación de la biodiversidad, p. 69-78. In: F. Martín-Piera, J.J. Morrone, A. Melic (Eds). Hacia um Proyecto CYTED Para el Inventario de la Diversidad Entomológica en Iberoamérica: Pribes 2000. Zaragoza, Sociedad Entomológica Aragonesa, 326p.

Morrone, J.J. \& J.V. CrisCI. 1995. Historical biogeography: introduction to methods. Ann. Rev. Ecol. Syst. 26: 373-401.

Morrone, J.J. \& C. Lopretto. 1995. Parsimony analysis of endemicity of freshwater Decapoda (Crustacea: Malacostraca) from southern South America. Neotropica 41 (105-106): 3-8.

MYers, A.A. 1991. How did Hawaii accumulate its biota? A test from the Amphipoda. Global Ecol. Biogeogr. Letts 1: 24-29.

Nelson, G. 1969. The problem of historical biogeography. Syst. Zool. 18: 243-246.

Nelson, G. \& N. Platnick. 1981. Systematics and biogeography: cladistics and vicariance. New York: Columbia Univ. Press, XI+567p.

Platnick, N.I. 1991. On areas of endemism. Aust. Syst. Bot., Collingwood, 4: 11-12.

Platnick, N.I. \& G. Nelson. 1978. A method of analysis for historical biogeography. Syst. Zool. 27: 1-16.

PosadAs, P. 1996. Distributional patterns of vascular plants in Tierra del Fuego: a study applying Parsimony Analysis of Endemicity (PAE). Biogeographica 72: 161-177.

RoN, S.R. 2000. Biogeografic area relationships of lowland Neotropical rainforest based on raw distributions of vertebrate groups. Biol. Jour. Linn. Soc. 71: 379-402.

Rosen, B.R. \& A.B. Smith. 1988. Tectonics from fossils? Analysis of reef-coral and sea-urchin distribution from late Cretaceous to Recent, using a new method. Gondwana and Tethys, p. 275-305. In: M.G. Audley-Charles \& A. Hallam (Eds). Geological Society Special Publication 37. Oxford, Clarendon Press, 317p.

Rosen, B.R. 1988. From fossils to earth history: applied historical biogeography, p. 437-481. In: A.A. MYERS \& P.S. GILLERS (Eds). Analytical Biogeography. London, Chapman \& Hall, 578p.

Silva, J.M.C. \& D.C. OREN. 1996. Application of parsimony analysis of endemicity in Amazonian biogeography: an example with primates. Biol. Jour. Linn. Soc. 59: 427-437.

Vega, I.L.; O.A. Ayala; D.E. Organista \& J.J. Morrone. 1999. Historical relationships of the Mexican cloud forests: a preliminary vicariance model applying Parsimony Analysis of Endemicity to vascular plant taxa. Jour. Biogeogr. 26: 1299-1305.

Recebido em 05.IX.2002; aceito em 29.XI.2002. 